\title{
Duration of Protection and Age-Dependence of the Effects of the SPf66 Malaria Vaccine in African Children Exposed to Intense Transmission of Plasmodium falciparum
}

\author{
Pedro L. Alonso, Tom A. Smith, \\ Joanna R. M. Armstrong-Schellenberg, Andrew Y. Kitua, \\ Honorathi Masanja, Richard Hayes, Nicole Hurt, \\ Fidel Font, Clara Menendez, Wencislaus L. Kilama, \\ and Marcel Tanner
}

\author{
Unidad de Epidemiologia y Bioestadistica, Hospital Clinic. Barcelona, \\ Spain; Department of Public Health and Epidemiology. Swiss Tropical \\ Institule, Basel, Switzerland; Tropical Health Epidemiology Unit, \\ London School of Hygiene and Tropical Medicine, London. \\ United Kingdom: Ifakara Centre and NIMR Headquarters, \\ National Institute for Medical Research, Ifakara, Tanzania
}

\begin{abstract}
The SPf66 synthetic vaccine is safe and partly efficacious against Plasmodium falciparum malaria among children 1-5 years old. The estimated vaccine efficacy [VE] for all clinical episodes over a period of 18 months after the third dose is $25 \%$ (95\% confidence interval $[\mathrm{CI}], 1 \%-44 \% ; P=.044)$. The observed temporal variations in efficacy could have been due to chance (likelihood ratio $\boldsymbol{X}^{2}=$ $13.8,8 d f ; P=.086)$. Efficacy against clinical malaria did not vary significantly with age $\left(\chi^{2}=1.07\right.$, $4 d f ; P=.90)$. Overall parasite density was $21 \%$ lower in vaccine recipients than in the placebo group (95\% CI, 0\%-38\%; $P=.044$ ). Further development of SPf66 may require trials to evaluate safety, immunogenicity, and efficacy when administered in the first year of life, together with other vaccines contained in the Expanded Programme of Immunization schedule.
\end{abstract}

Malaria, especially that caused by Plasmodium falciparum, is the most important parasitic disease of humans. Estimates of the burden of disease and death are imprecise but include $>300$ million clinical episodes and 1-3 million deaths per year. Even though malaria has a wide geographic distribution and is endemic in $>100$ countries, $>90 \%$ of the world's burden of discase and death concentrates among young children and primigravid pregnant women living in sub-Saharan Africa.

Development of vaccines against $P$. falciparum offers multiple potential targets in all stages of the parasite's life cycle, and technological developments allow for the possibility of combining antigens from either one or several stages. Each target or combination of targets may possibly imply different outcomes. In the absence of a pre--erythrocytic stage vaccine that induces stcrilizing lifelong immunity (clearly the most desirable option), vaccines derived from asexual-blood stage

Received 27 November 1995; revised 22 April 1996.

The protocol was reviewed and approved by the ethical committecs of al! participating institutions, the World Health Organization, and the Tanzanian Commission for Science and Tcchnology (UTAFITI NSR/RCA 90). Detailed written informed consent was obtained from the parents and guardians of children.

Financial support: Major financial support was provided by the participating institutions. F.F. is supported by Medicus Mundi through a grant from the Spanish Agency for International Cooperation (AECL). J.R.M.A.-S. and R.H. are partly supported by UK Medical Research Council. The Ifakara Centre receives major financial support from the Swiss Development Cooptration, AEC I, and the Swiss Tropical Institute. This investigation received financial support from the UNDP/World Bank/WHO Special Programme for Research and Training in Tropical Diseases.

Reprints or correspondence: Dr. P. L. Alonso, Unidad de Epidemiologia y Bioestadistica, Hospital Clinic, Villarroel 170, E-08036 Barcelona, Spain.

The Journal of Infectious Diseases 1996;174:367-72

(10) 1996 by The University of Chicago. All rights reserved.

$0022 \quad 1899 / 96 / 7402-0018 \$ 01.00$ antigens may be of particular interest in Africa, because they may mimic the development of natural anti-parasite and clinical immunity in children living in endemic areas [1].

SPf66 is a synthetic hybrid polymer, solubilized in saline solution and adsorbed onto aluminium hydroxide. The monomer unit is a chemically synthesized peptide of 45 amino acids that contains sequences derived from three asexual-blood stage proteins $(83,55$, and $35 \mathrm{kDa})$ linked by repeat sequences from the circumsporozoite protein of the $P$. falciparum parasite [2]. SPf66 has been shown to be safe and partially protective against $P$. falciparum malaria in populations $>1$ year of age living in low-endemicity areas of South America [3, 4, 5]. In the La Tola trial in Colombia [3], SPf66 was cfficacious for at least 1 year after the third dose, and there was a suggestion that efficacy was higher among young children and older adults. However, all of the effects of SPf66, including safety, immunogenicity, and short- and long-term efficacy, could vary with different transmission levels.

The first safety, immunogenicity, and efficacy results of SPf66 from Africa came from a trial in the Kilombero Valley, an area of intense perennial transmission of $P$. falciparum in southern Tanzania $[6,7]$. In the study, the efficacy analysis of the SPf66 vaccine in 1- to 5-year-old children indicated that three doses reduced the risk of first or only episodes of clinical malaria by $31 \%$ ( $95 \%$ confidence interval [CI], $0 \%-52 \% ; P$ $=.045$ ) [7]. Clinical malaria was defined as fever (axillary temperature $\geqslant 37.5^{\circ} \mathrm{C}$ ) with parasite density $>20,000 / \mu \mathrm{L}$. The efficacy against all episodes of fever and parasitemia (a less specific case definition of clinical malaria), though lower than that against hyperparasitemic episodes, was $25 \%$ (95\% CI, $2 \%-$ $43 \% ; P=.03$ ). Thus, the vaccine had partial efficacy against clinical malaria during 1 year following administration of the third dose. Longer-term protection remained to be established, 
including a theoretically possible rebound in morbidity among vaccine recipients if immunity induced by the vaccine decreased over time. More recently, a trial among nonimmune Gambian infants, who were 6-to 11 -months old at the time of the first dose, has failed to document significant levels of protection (vaccine efficacy $[\mathrm{VE}]=8 \% ; 95 \% \mathrm{CI},-18 \%-$ $29 \%$ ) [8].

Here we report further analyses of the data from the Kilombero SPf66 trial. We examine whether vaccine efficacy extends beyond the first year of follow-up, whether there is evidence to suggest that efficacy varies with age, and the effect of the vaccine on parasite density in more detail.

\section{Methods}

Study area and period. The study was done in the village of Idete in the Kilombero river plain in southern Tanzania. Details of the study area and study design have been presented elsewhere $[9,10]$. In brief, the Kilombero Valley is an area of intense and perennial malaria transmission. Most inhabitants are subsistence farmers growing maize and natural irrigation rice. The Kilombero SPf66 vaccine trial was a randomized double-blind, placebo-controlled, community-based efficacy trial. After screening procedures were completed, 586 children aged $1-5$ years received three doses of either SPf66 or placebo.

The main analyses presented here are based on the period starting 4 weeks after the administration of the third dose of vaccine (September 1993). The previously reported analyses [7] considered follow-up for 48 weeks, until the end of July 1994. We now describe results including an extended follow-up for a further 26 weeks, terminating on 31 January 1995. Although the randomization code was broken in early August 1994 for the analysis, the study population and ficld team remained blind for the extra period of follow-up, while further supplies of SPf66 were prepared and transported to Tanzania. In the first week of February 1995, SPf66 was offered to children who had previously received placebo, as agreed between the village and the investigators at the beginning of the trial.

Parasitologic and clinical surveillance. Cross-sectional surveys, at which blood films for parasitologic examination were taken, were done on a group of 219 children during weeks 30,32 , $34,38,50,73$, and 99 of the follow-up $(4,6,8,12,24,47$, and 73 weeks, respectively, after the third dose). Similar surveys were also done between the second and third doses on a different subgroup of 176 children at weeks 8, 10, 12, 16, and 24. All 586 children making up the complete study cohorts were seen at a baseline survey 2 weeks before receiving the first dose [10]. Before each dose of either vaccine or placebo, all study children received one dose of sulfadoxine-pyrimethamine $(25 \mathrm{mg}$ of sulfadoxine and $0.75 \mathrm{mg}$ of pyrimethamine/kg of body weight).

Passive case detection of clinical episodes was done at the only village dispensary as described $[7,10]$. In brief, each patient attending the dispensary for a perceived illness was screened by project medical personnel, who provided $24-\mathrm{h}$ coverage. Children were identified using the census and individual identification cards, and axillary temperatures were checked with an electronic thermometer (MBO, Munich). If the temperature was $\geqslant 37.5^{\circ} \mathrm{C}$, a fin- gerprick blood sample was collected and thick and thin blood films prepared. Children were then cared for by the routine medical services at the dispensary. Clinically suspected malaria episodes were treated, following national standard guidclines, with $25 \mathrm{mg} /$ $\mathrm{kg}$ chloroquine.

Statistical methods. Analysis of risk of clinical malaria considered all episodes satisfying the primary case definition $(>20,000$ parasites $/ \mu \mathrm{L}$ and an axillary temperature $\geqslant 37.5^{\circ} \mathrm{C}$ ). Note that this differs from previous analysis [7], which were restricted to first or only episodes. For the present analyses, time at risk was computed in weeks. Any week that included days at risk was included. To avoid the inclusion of treatment failures and recrudescence as repeated events, second events $<28$ days after an episode wcre excluded. Children were not considered at risk for those 28 days.

The proportion of the weeks at risk in which an episode occurred was analyzed by logistic regression using the EGRET software package [11]. Because there were small imbalances between vaccine and placebo groups with respect to age and distance from the dispensary [7], all analyses allowed for these factors as defined in the analytical plan [7]. Analysis also considered effects of vaccination status and of time periods of $\sim 8$ weeks. The agc-dependence of the effect of vaccination was tested by including a term representing age for vaccine recipients and 0 for placebo recipients. Changes in vaccine efficacy over time were tested similarly.

The above analyses assume that multiple episodes in the same child occur independently of each other. To test whether this assumption affected the conclusions, a logistic-normal regression model [12] was fitted, which allowed for variation in susceptibility between children. Adjusting for this potential source of variation did not improve the fit of the model, and we therefore report tests and CIs derived from the original analysis.

Parasite positivity was analyzed by logistic regression in relation to age group, vaccination status, and time period of the survey. Allowance for variation between children in susceptibility again did not improve the fit of the model.

Analyses of parasite densities were related to vaccination status, survey, and age using least squares regression models. Aging of the cohort meant that the age distribution was different at each survey. Age-specific average parasite densities allowing for survey variation were computed by the method of least squares means [13]. The same method was used to calculate survey-specific average adjusted for age.

Parasite densities on successive slides from the same child are correlated. Models that allowed for this were fitted by residual maximum likelihood [14] using the SAS MIXED procedure [15]. Statistical significance test was determined by likelihood ratio (LR) tests.

\section{Results}

Clinical episodes. During the additional 6 months of follow-up from 1 August 1994 to 31 January 1995, there were no deaths among cohort children. Twenty-nine episodes of clinical malaria satisfying the primary case definition (10 vaccine and 19 placebo) were detected through passive case detection. Between 4 weeks after the third dose and the end of follow-up 18 months later, a total of 121 clinical episodes werc 


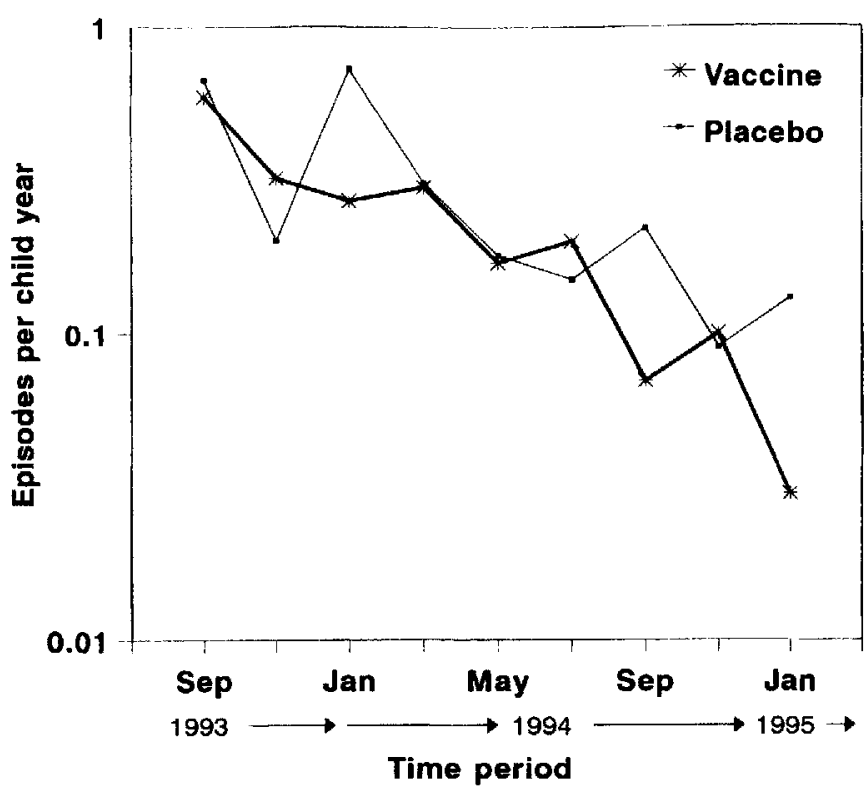

Figure 1. Incidence of clinical episodes among SPf66 vaccine and placebo cohorts after third dose by 8 -week time period.

recorded in placebo recipients $(0.30$ episodes/child-year) and 83 in SPf66 recipients ( 0.23 episodes/child-year). The age- and distance-adjusted estimate of vaccine efficacy was $25 \%(95 \%$ CI, $1 \%-44 \% ;$ LR $\left.\chi^{2}=4.04,1 d f ; P=.044\right)$. The incidence of clinical episodes, among both vaccine and placebo recipients, showed a decline with time (figure 1). Age-standardized incidence estimates indicated that the decline in incidence over the period of follow-up could not be entirely accounted for by the aging of the cohort (age- and distance-adjusted tests of the difference in risk between 2-month time periods, LR $\chi^{2}=$ $43.4,8 d f ; P<.001)$. Although vaccine efficacy appeared to vary over time and to be restricted to several periods (figure 1), there was no clear trend, and these variations could have bcen due to chance (test of heterogeneity in efficacy between time periods, LR $\left.\chi^{2}=13.8,8 d f ; P=.086\right)$.

Risk of clinical malaria decreased steeply with age (table 1 and figurc 2). Incidence of clinical malaria was lower among vaccine recipients than among placebo rccipients in all age groups. The corresponding vaccine efficacy estimates by age are also presented in table 1 . There was no evidence of vaccine efficacy changing with age (LR test for heterogeneity, $\chi^{2}=$ 1.07, $4 d f ; P=.90 ;$ LR test for trend, $\chi^{2}=0.26,1 d f ; P=$ $.69)$. In children $<2.5$ years of age, the vaccine efficacy of $29 \%$ corresponds to a time period -standardized reduction in risk from 0.92 to 0.65 episodes per child-year (i.e., a substantial reduction in risk of 0.27 prevented episodes per child-year). The corresponding reduction in children $>5$ years old, for whom the estimated VE was $43 \%$, was from 0.12 to 0.07 episodes per child-year ( 0.05 prevented cpisodes).

Parasite positivity. Treatment with sulfadoxine-pyrimethamine before each dose of vaccine aimed to optimize the re- sponse to the vaccine. It consequently also ensured that followup began with a low prevalence of parasitemia in both vaccine and placebo groups (figure 3). Prevalence increased rapidly with time, gradually tending toward levels similar to that at screening $(92 \%)$. The difference between overall parasite positivity in vaccine and placebo groups was small and not statistically significant (LR $\chi^{2}=2.25,1 d f ; P=.13$ ). The prevalence of patent parasitemia averaged over all surveys declined with age (LR $\left.\chi^{2}=16.74,4 d f ; P=.002\right)$, but this decline was not very steep. There was no evidence that the effect of vaccination varied with age ( $\mathrm{LR} \chi^{2}=0.26,1 d f ; P=.6$ ).

Parasite densities. Overall, parasite density after the third dose was $21 \%$ lower in vaccine recipients than in the placebo group $(95 \% \mathrm{CI}: 0 \%-38 \% ; F$ statistic $=4.06,1740 \mathrm{df} ; P=$ .044). Parasite densities decreased with age, both after the third dose (figure $4 \mathrm{~A}$, table 2 ) and between the second and third doses (figure 4B). Allowance for differences between surveys did not account for the age trends (LR test for effect of age adjusted for survey, $\left.\chi^{2}=28.16,4 d f ; P<.001\right)$. After the third dose, parasite density was much more strongly age-dependent among vaccine recipients than among placebo recipients. In the youngest age group, the densities in children receiving three doses of vaccine were higher than in placebo recipients, but the reverse was true for older children. The interaction between a linear effect of age and vaccination status was statistically highly significant (LR $\chi^{2}=12.6,1 d f ; P<.001$ ).

\section{Discussion}

Eighteen months after the third dose of SPf66 was given in the Kilombero trial, there is evidence to suggest that vaccine efficacy against clinical malaria was sustained. Previous analyses of this trial indicated that the vaccine is safe and immunogenic and reduced the risk of first or only episodes of clinical malaria by a best estimate of $31 \%$ during a 1 -year period of follow-up after the third dose. By including a further 6 months of follow-up, as well as all malaria episodes and not just first or only episodes, we estimate vaccine efficacy to be $25 \%(95 \%$ $\mathrm{CI}, 1 \%-44 \%$ ). This figure therefore represents the reduction in the burden of malaria attributable to vaccination in this group of children over a period of 18 months after the third dose.

None of the published trials were designed to look at variations in efficacy over time, and observed data must be interpreted with caution. The La Tola trial [3] suggested some degree of variation in vaccine efficacy over time. However, there was evidence of efficacy in all time periods and a suggestion that efficacy was highest in the first and last 3-month period. The apparent temporal variations in the trial of Las Majadas in Venezucla [4] are very difficult to interpret given the nonrandomized nature of the trial.

In the Tanzania trial (figure 1), there is a suggestion that vaccinc efficacy has been limited to certain time periods (January and September 1994 and January 1995). Although a number of hypotheses related to transmission intensity or seasonal cir- 
Table 1. Annual incidence of clinical episodes by age group among SPf 66 vaccine and placebo recipients and corresponding vaccine efficacy cstimates.

\begin{tabular}{|c|c|c|c|c|c|c|c|}
\hline \multirow[b]{2}{*}{$\begin{array}{l}\text { Age, } \\
\text { months }\end{array}$} & \multicolumn{3}{|c|}{ Vaccine } & \multicolumn{3}{|c|}{ Place bo } & \multirow[b]{2}{*}{$\begin{array}{c}\text { Vaccine efficacy. } \% \\
(95 \% \text { confidence interval })\end{array}$} \\
\hline & $\begin{array}{l}\text { No. of } \\
\text { episodes }\end{array}$ & $\begin{array}{l}\text { Wceks } \\
\text { at risk }\end{array}$ & $\begin{array}{c}\text { Adjustd } \\
\text { incidence } \\
\text { rate }^{*}\end{array}$ & $\begin{array}{l}\text { No. of } \\
\text { episodes }\end{array}$ & $\begin{array}{l}\text { Wecks } \\
\text { at risk }\end{array}$ & $\begin{array}{c}\text { Adjusted } \\
\text { incidence } \\
\text { ratc* }\end{array}$ & \\
\hline$<30$ & 24 & 1508 & 0.65 & 27 & 1220 & 0.92 & $29(-24,59)$ \\
\hline $30-35$ & 20 & 1900 & 0.56 & 24 & 1793 & 0.62 & $9(-66,50)$ \\
\hline $36 \cdots 47$ & 19 & 3843 & 0.30 & 29 & 4653 & 0.31 & $5(-70,47)$ \\
\hline $48-59$ & 11 & 3557 & 0.17 & 20 & 4021 & 0.25 & $32(-42,68)$ \\
\hline$\geqslant 60$ & 9 & 7765 & 0.07 & 21 & 9145 & 0.12 & $43(-25,74)$ \\
\hline
\end{tabular}

* Estimated incidence for fifth 8 -week period of follow-up, adjusted for distance from dispensary.

culation of certain parasite strains could be postulated, the apparent variations in vaccine efficacy over time may have been simply due to chance. Nonetheless, one of those periods when differences between vaccine and placebo recipients appear to be more noticeable is precisely at the end of the followup. This would support the suggestion that the vaccine remains efficacious for at least 18 months after the third dose. It would also argue against decreasing vaccine efficacy or rebound in morbidity among vaccine recipients.

The data also highlight the difficulty of establishing longterm protection afforded by an asexual-stage vaccine in an area of high transmission where the age pattern of disease is strongly shifted toward the younger age groups. Indecd, whilc children $<30$ months old had nearly 1 clinical episode detected through passive case detection every year, children $>60$ months old had just over 0.1 episodes per year. Other authors have argued that even in areas of very high transmission, there are relatively

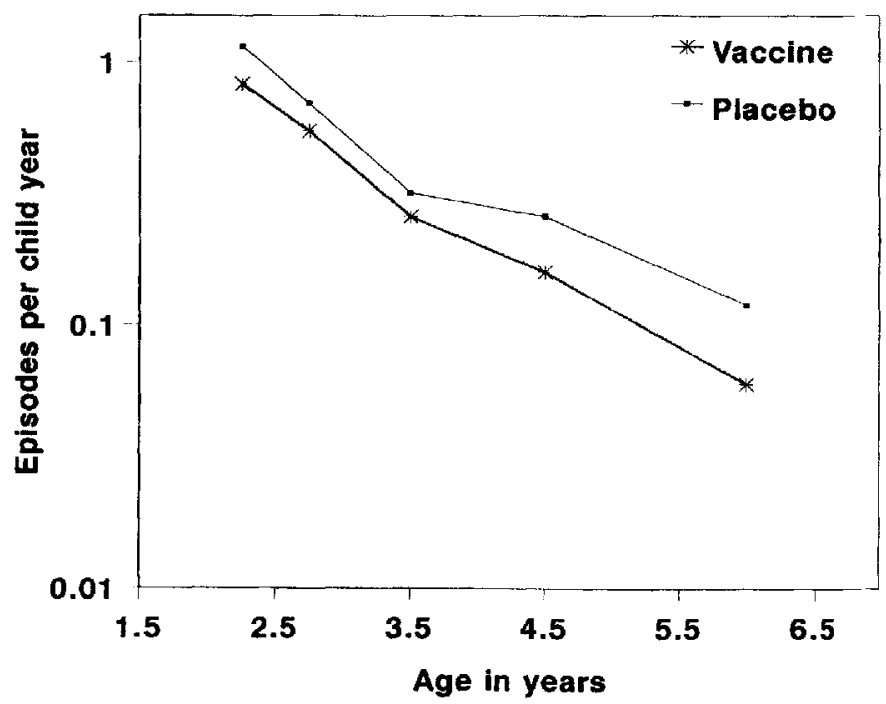

Figure 2. Incidence of clinical episodes among SPf66 vaccine and placebo recipients after third dose by age at time of episode. small differences in the incidence of malaria among children aged $0-6$ years and subject to a very intense and active followup [16]. These children have, on average, 4 episodes per year. While this may be truc, our data clearly show that the risk of clinical malaria cases that attend the dispensary and fulfill a definition of clinical malaria with a high specificity decreases steeply with age in this area of high and perennial transmission. As previously argued [17], it may be that the cases seen at the dispensary and therefore ascertained through passive case detection represent the more severe episodes in the clinical spectrum of malaria, while many other mild, self-limited, nonsevere cases go unreported.

The trial was not designed, and therefore had little power, to demonstrate differences in vaccine efficacy between age groups. In any case, the data suggest that clinical efficacy is not restricted to children of any specific age group or that the efficacy depends on age. Younger children seem to be protected

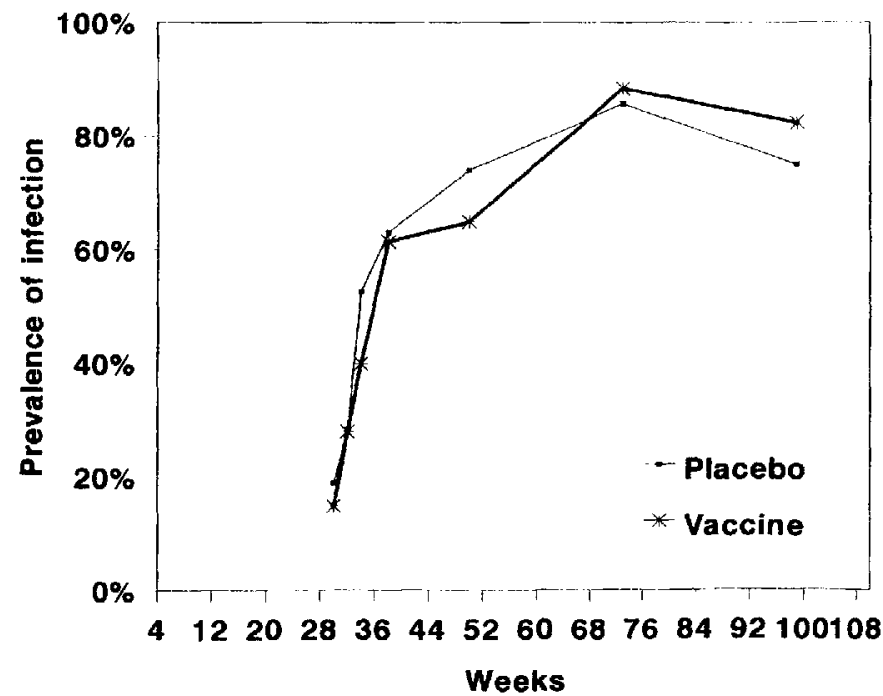

Figure 3. Prevalence of parasitemia by time period among SPf66 vaccine and placebo recipients. 

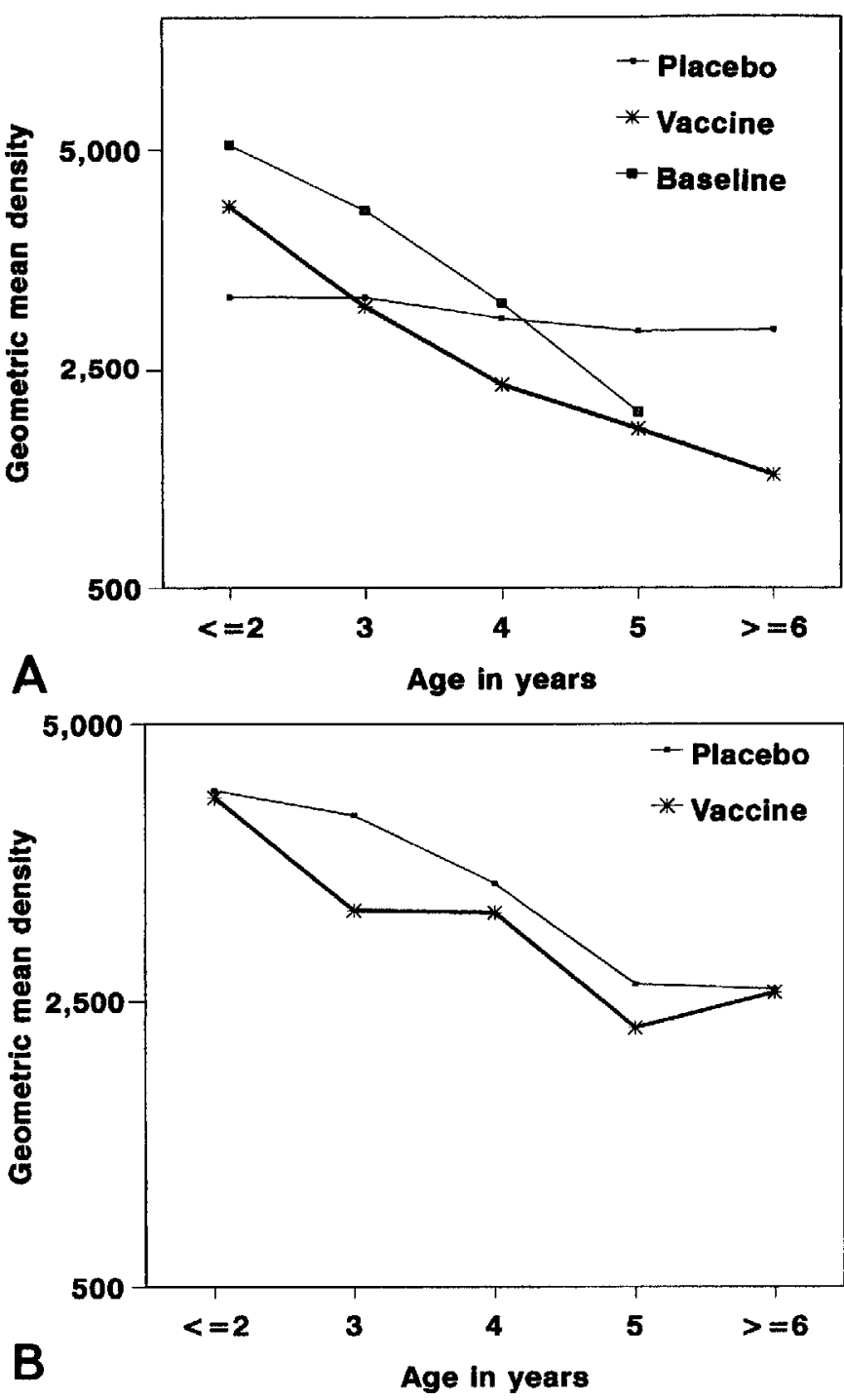

Figure 4. Geometric mean parasite densities by age at time of episode after third dose of SPf66 vaccine (A) and between second and third dose (B).

to a similar degree as older ones. The impact of even a modest efficacy of $30 \%$ in the younger age groups is large, given the high incidence of malaria in this age group, and corresponds to 0.27 prevented episodes per child-year.

A primary effect of SPf66 on asexual-stage parasites was anticipated because the vaccine includes amino acid sequences derived from merozoite membrane proteins. In the original artificial challenge experiments in Colombia in both monkeys and humans [18, 19], self-limiting symptomatic infections were observed in some immunized individuals, implying that a primary effect of SPf66 may be to reduce densities of blood-stage parasites. In our trial, there is evidence of the vaccine reducing parasite densities after the third dose by $\sim 20 \%$.

When comparing parasite densities between SPf66 and placebo recipients in different age groups, it appears that threc doses of vaccine were associated with a reduction in parasite densities in the older children and with an apparent negative effect in the youngest children. However, the comparison of the age-dependence of densities after the third dose with those in the baseline survey (figure 4A) and between the second and third dose suggests that rather than the vaccine group showing an unusually strong relationship between age and density, the placebo group shows an unexpectedly weak age-dependence of densities. The reason for this temporary decrease in agedependence is unclear. It may be a consequence of repeated sulfadoxine-pyrimethamine treatment. This resulted in an extended period of reduced antigenic stimulation in the placebo group at the same time as the vaccine group was being immunized with SPf66. However, this may help explain the lower parasite densities seen in the young children receiving placebo but can hardly explain the higher parasite densities in older children in the placebo group compared to baseline data.

Recently reported results from a trial of SPf66 among nonimmune Gambian infants 6- to 11-months old at the time of first dose have not documented significant levels of protection [8]. The results raise further questions on the potential public health relevance of the vaccine. Efficacy estimates were based on a subgroup analysis of the study children and a short 3.5-month period of follow-up. The authors suggest that the lack of a significant level of protection documented in the SPf66 trial in Gambia may have been due to the short follow-up and that it may take the vaccine this long to show any apparent effect. While this may be true, data on this point are lacking.

Some of the differences in the estimated efficacies between the trial in Gambia and all other trials may include the design and its emphasis on active case detection, the ages and level of preimmunity of the study children, and the specificity of the case definitions of the primary end point. It is also possible that the apparently contradictory results between Gambia and all other sites may have been due to chance, as the upper 95\% confidence bound overlaps with the lower bound from the Tanzanian trial. These results also highlight the need for adequate coordination of trials that ensure complementarity and comparability.

The benefit of any malaria vaccine in sub-Saharan Africa will depend mainly on its effectiveness in preventing severe disease and death. Its applicability is likely to depend on the feasibility of delivering it through the Expanded Programme of Immunization. Recent estimates suggest that a vaccine with a $30 \%$ efficacy against death may be a highly cost-effective public health intervention in countries of sub-Saharan Africa in which malaria is endemic if it can be delivered through the existing Expanded Programme of Immunization (Evans D, personal communication). A further large-scale study to investigate these questions and complete the process of vaccine development for SPf66 is required [20]. Also, further work to understand the reason for the different estimates of efficacy between different trial sites is needed. The comprehensive approach to all these questions as well as a systematic review of 
Table 2. Geometric mean parasite densities by age group and treatment group between second and third dose and after third dose of either vaccine or placebo.

\begin{tabular}{|c|c|c|c|c|c|c|c|}
\hline \multirow[b]{2}{*}{$\begin{array}{l}\text { Time point, } \\
\text { age (years) }\end{array}$} & \multicolumn{2}{|c|}{ Vaccine } & \multicolumn{2}{|c|}{ Placebo } & \multirow[b]{2}{*}{$\begin{array}{l}\text { Lower } 95 \% \\
\text { confidence limit* }\end{array}$} & \multirow[b]{2}{*}{$\begin{array}{l}\text { Ratio of } \\
\text { densities* }\end{array}$} & \multirow[b]{2}{*}{$\begin{array}{c}\text { Upper } 95 \% \\
\text { confidence limit* }\end{array}$} \\
\hline & $n$ & $\begin{array}{l}\text { Parasite } \\
\text { density }\end{array}$ & $n$ & $\begin{array}{l}\text { Parasite } \\
\text { density }\end{array}$ & & & \\
\hline \multicolumn{8}{|c|}{ Between second and third dose } \\
\hline$\leqslant 2$ & 80 & 3708 & 112 & 3817 & 0.56 & 0.97 & 1.69 \\
\hline 3 & 42 & 2334 & 62 & 3449 & 0.34 & 0.68 & 1.36 \\
\hline 4 & 55 & 2315 & 62 & 2608 & 0.49 & 0.91 & 1.68 \\
\hline 5 & 40 & 1441 & 52 & 1725 & 0.42 & 0.82 & 1.62 \\
\hline$\equiv 6$ & 14 & 1666 & 19 & 1689 & 0.34 & 1.00 & 2.90 \\
\hline \multicolumn{8}{|c|}{ After third dose } \\
\hline$\leqslant 2$ & 100 & 3699 & 74 & 2288 & 0.98 & 1.67 & 2.84 \\
\hline 3 & 77 & 2174 & 92 & 2284 & 0.50 & 0.82 & 1.32 \\
\hline 4 & 72 & 1443 & 83 & 2046 & 0.41 & 0.69 & 1.14 \\
\hline 5 & 52 & 1146 & 54 & 1910 & 0.29 & 0.54 & 1.00 \\
\hline$\geqslant 6$ & 61 & 904 & 88 & 1930 & 0.28 & 0.45 & 0.73 \\
\hline
\end{tabular}

* Allowing for differences between surveys in average densities.

all published and ongoing work on this vaccine will clarify the potential of SPf66 as a potential public health tool and help in the process of development of new vaccines with increased efficacy.

\section{Acknowledgments}

We thank the children and their parents, the dispensary staff, and the whole village of Idete. Special thanks go to the staff of the Ifakara Centre and the district authorities. Research clearance was granted by the Tanzanian Commission for Science and Technology (UTAFITI NSR/RCA 90). We are grateful to M. E. Patarroyo for providing the SPf66 peptide and encouraging the execution of the trial.

\section{References}

1. Alonso PL, Molyneux ME, Smith T. Design and methodology of fieldbased intervention trials of malaria vaccines. Parasitol Today 1995; 11 : $197-200$

2. Moreno A, Patarroyo ME. Development of an asexual blood stage malaria vaccine. Blood 1989;74:537-46.

3. Valero MV, Amador LR, Galindo C, et al. Vaccination with SPf66, a chemically synthesized vaccine, against Plasmodium falciparum malaria in Colombia. Lancet 1993;341:705-10.

4. Noya O, Gabaldon BY, Alarcon de Noya B, et al. A population-based clinical trial with the SPf66 synthetic Plasmodium falciparum malaria vaccine in Venezucla. I Infect Dis 1994; 170:396-402.

5. Sempertegui F, Estrella B, Moscoso F, et al. Safety, immunogenicity, and protective effect of the SPf66 malaria synthetic vaccine against Plasmodium falciparum infection in a randomized double blind placebo controlled ficld trial in an endemic area of Ecuador. Vaccine 1994; 12 : $337-42$.

6. Teuscher T, Armstrong-Schellenberg JRM, Bastos de Azevedo I, et al. SPf66, a chemically synthesized subunit malaria vaccine, is safe and immunogenic in Tanzanians exposed to intense malaria transmission. Vaccine $1994 ; 12: 328-36$

7. Alonso PL, Smith T, Armstrong-Schellenberg JRM, et al. Randomised trial of efficacy of SPf66 vaceine against Plasmodium falciparzm malaria in children in children in southern Tanzania. Lancet 1994;343:1175-81.

8. D'Alessandro $U$, Leach $A$, Drakeley $\mathrm{CJ}$, et al. Efficacy trial of malaria vaccine SPf66 in Gambian infants. Lancet 1995;346:462 7 .

9. Tanner M, De Savigny D, Mayombana C, et al. Morbidity and mortality at Kilombero, Tanzania, 1982-1988. In: Feachem RG, Jamison DT, cds. Disease and mortality in sub-Saharan A frica. Oxford: Oxford University Press, 1991:286-305.

10. Alonso PL, Tanner M, Smith T, et al. A trial of the synthetic malariat vaccine SPf66 in Tanzania: rationale and design. Vaccine 1994;12: $181-6$.

11. Statistics and Epidemiology Research Corporation. EGRET: User's manual. Seattle: SERC, 1991

12. Pierce DA, Sands BR. Extra-Bemouilli variation in binary data Corvallis, OR: Department of Statistics, Oregon State University, 1975; technical report no. 46.

13. Searle SR, Speed FM, Milliken GA. Population marginal means in the linear model: an alternative to least squares means. Am Statistician $1980 ; 34: 216-21$.

14. Patterson HD, Thompson R. Recovery of inter-block information when block sizes are unequal. Biometrika 1974; 58:545 54.

15. SAS Institute. SAS/STAT software: changes and enhancements, release 6.07. Cary, NC: SAS Institute, 1992; SAS technical report no. P-229.

16. Trape JF, Rogier C. Efficacy of SPf66 vaccine against malaria in children. Lancet $1995 ; 345 ; 134$.

17. Alonso PL, Smith T, Tanner M. Efficacy of SPf66 vaccinc against Plasmodium falciparum malaria in childres. Lancet 1995;345:135.

18. Patarroyo ME, Romero P, Torres MI, et al. Induction of protective immunity against experimental infection with malaria using synthetic peptides. Nature 1987; 328:629 32.

19. Patarroyo ME, Amador R, Clavijo P, et al. A synthetic vaccine protects humans against challenge with asexual blood stages of Plasmodium falciparum. Nature 1988;332:158-61.

20. Tanner M, Teuscher T, Alonso PL. The first malaria vaccine. Parasitol Today $1995 ; 11: 10-3$. 